\title{
Estimating the uncertainty of the liquid mass flow using the orifice plate
}

\author{
Anna Golijanek-Jędrzejczyk ${ }^{1, *}$, Dariusz Świsulski ${ }^{1}$, Robert Hanus $^{2},{\text { Marcin } Z \mathrm{Zych}^{3} \text {, and Leszek Petryka }}^{4}$ \\ ${ }^{1}$ Gdańsk University of Technology, Faculty of Electrical and Control Engineering, 80-233 Gdańsk, Poland \\ ${ }^{2}$ Rzeszów University of Technology, Faculty of Electrical and Computer Engineering, 35-959 Rzeszów, Poland \\ ${ }^{3}$ AGH University of Science and Technology, Faculty of Geology, Geophysics and Environmental Protection, 30-059 Kraków, Poland \\ ${ }^{4}$ AGH University of Science and Technology, Faculty of Physics and Applied Computer Science, 30-059 Kraków, Poland
}

\begin{abstract}
The article presents estimation of measurement uncertainty of a liquid mass flow using the orifice plate. This subject is essential because of the widespread use of this type of flow meters, which renders not only the quantitative estimation but also qualitative results of this type so those measurements are important. To achieve this goal, the authors of the paper propose to use the theory of uncertainty. The article shows the analysis of the measurement uncertainty using two methods: one based on the "Guide to the expression of uncertainty in measurement" (GUM) of the International Organization for Standardization with the use of the law of propagation of uncertainty, and the second one using the Monte Carlo numerical method. The paper presents a comparative analysis of the results obtained with both of these methods.
\end{abstract}

\section{Introduction}

The knowledge of the accuracy of the mass flow of liquids is significant due to the fact that it occur in most processes and is a significant research topic. Especially experimental or particularly accredited laboratories should focus on full and correct estimation of measurement uncertainty of mass flow.

Modern metrology [1,2] requires that in addition to the quantitative measure of the particular value, its quality should be given as well - preferably the uncertainty of measurement. For this reason, in each thematic area, both in the measurement of power $[3,4]$, biomedical [5], and electric values [6,7], an estimate of the uncertainty of the measured value is provided.

This publication presents the methodology for estimating uncertainty of measurement of the mass flow of the liquid measured with the reducing flow meter. There are various methods and measuring instruments engaged to measure the mass flow [8-12] such as: orifice, classical Venturi reducer, thermoanemometer, Prandtl Pitot tube or the radiation methods [13]. Due to the nature of the analyzed issue these methods continue to be developed. The reducing flow meter was selected for analysis because of its most common application thanks to its simple structure, reliable operation and the usability in a wide range of pressures and temperatures of transported substances.

There is an available study addressing this significant issue of selecting an appropriate method [14]. However, this analysis is incomplete due to the lack of estimation of uncertainty of Type A.
The following paper presents the complete methodology for calculating the uncertainty of measurement of the mass flow of the liquid reducing flow meter using both: the analytical method [1] and Monte Carlo simulation - the numerical one [15].

The analytical method is based on a convolution of the input distribution values, using a mathematical model. In this case, the designated measure of uncertainty is the expanded uncertainty, calculated as the product of the expansion coefficient $k$ and the standard uncertainty value.

The numerical method is based on determining the uncertainty of measurement based on the extension range, which is determined by probability distribution of the measured value quantiles. The parameters of this distribution are: the expected value - as the estimate of the output value, the standard deviation and the confidence interval for a given probability level.

\section{The study}

The most common reducer of a stream is the orifice. It is a thin disc mounted in a pipe so that the axis of the bore coincides with the axis of the tube (Fig. 1).

The liquid stream flowing through the pipe becomes reduced ahead of the orifice. In contrast, the liquid stream insight the reducer reaches a minimum crosssection, and behind it the flow gradually expands to fill the entire volume of the pipe. The orifice fluid pressure increases slightly and is reduced to a minimum in the orifice at the narrowest flow cross-section. 


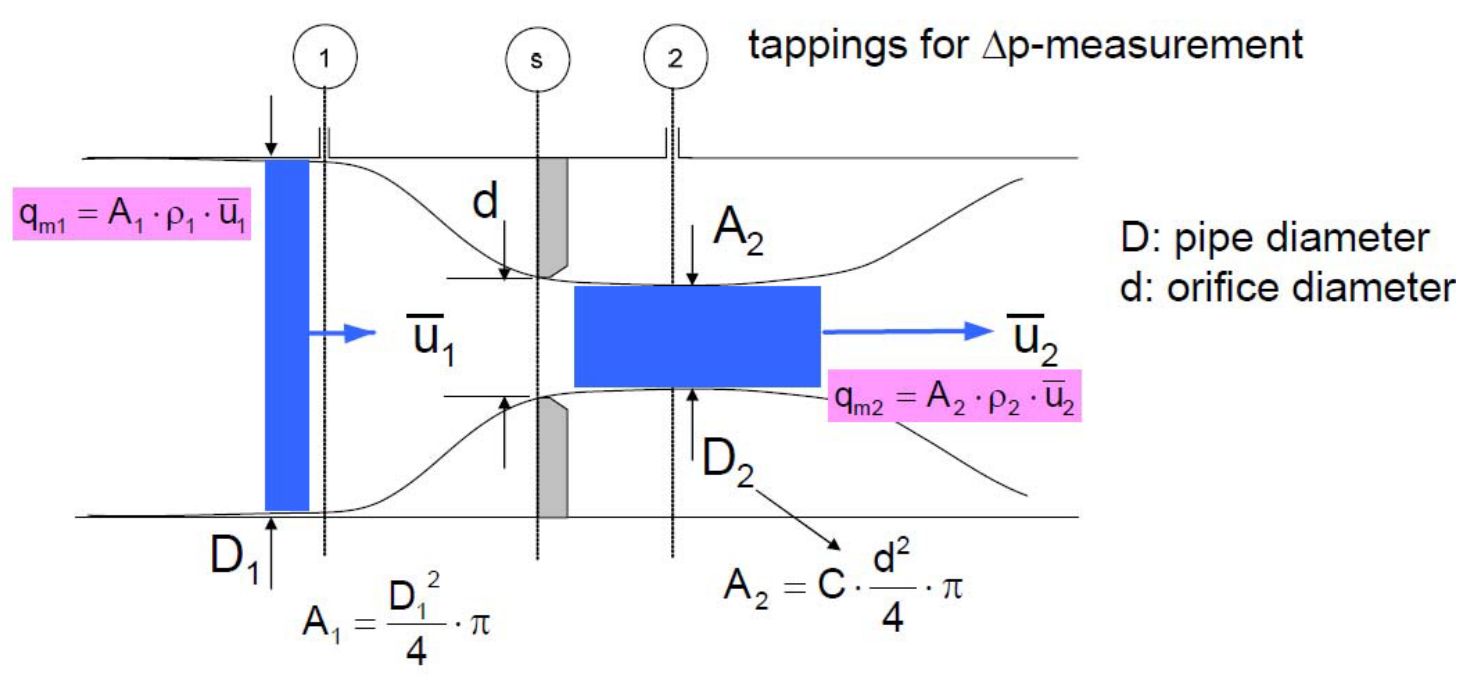

Fig. 1. Fluid flow through a measurement orifice [11].

The relation between the mass flow $q_{\mathrm{m}}[\mathrm{kg} / \mathrm{s}]$ and the pressure difference $\Delta p$ can be determined by the transformation of pressure energy into kinetic energy (speed) at a flow through the reducer. During this process, it is assumed that the continuity of the fluid stream is constant and its density does not change during the passage through different cross sections.

Those conditions allow the determination of the mass flow $q_{\mathrm{m}}$ as follows [2]:

$$
q_{m}=\frac{C}{\sqrt{1-\beta^{4}}} \varepsilon \frac{\pi}{4} d^{2} \sqrt{2 \Delta p \rho_{1}}
$$

where:

$C$ - a discharge coefficient [-],

$d$ - orifice diameter $[\mathrm{m}]$,

$\beta$-relation between orifice and pipe diameter:

$$
\beta=\frac{d}{D}
$$

$D$ - pipe diameter $[\mathrm{m}]$,

$\varepsilon$ - compressibility of different fluids. For water and other liquids $\varepsilon=1$.

$\Delta p$ - pressure difference [Pa], $\rho_{1}-$ density of medium $\left[\mathrm{kg} / \mathrm{m}^{3}\right]$.

When substituting equation (2) into (1):

$$
q_{m}=\frac{C}{\sqrt{1-\left(\frac{d}{D}\right)^{4}}} \varepsilon \frac{\pi}{4} d^{2} \sqrt{2 \Delta p \rho_{1}}
$$

The mass flow $q_{\mathrm{m}}$ is determined indirectly, and the function of the measurement is dependent on the following parameters $q_{\mathrm{m}}=f\left(C, d, D, \Delta p, \rho_{1}\right)$.
The analysis of the uncertainty of measurement of the mass flow $q_{\mathrm{m}}$ presented further in this publication was carried out under the following assumptions:

- the medium is a fluid, that is a ratio $\varepsilon=1$,

- at any point in any cross section perpendicular to the axis of the flow, a continuous stream of the fluid flow is maintained and equal to the speed,

- $\quad$ incompressible fluid without internal friction and a constant density $\rho_{1}=\rho_{2}=$ const.,

- a minimum of 30 measurements were made on the basis of which the $n=30$ observations of mass flow $q_{\mathrm{m}}$ were achieved.

\subsection{Analysis of the measurement uncertainty according to GUM}

Complex uncertainty $u_{\mathrm{c}}\left(q_{\mathrm{m}}\right)$ determining the mass flow $q_{\mathrm{m}}$ is defined as follows [1]:

$$
u_{c}\left(q_{m}\right)=\sqrt{u_{A}^{2}\left(q_{m}\right)+u_{B}^{2}\left(q_{m}\right)}
$$

where: $u_{A}\left(q_{m}\right)$ - uncertainty Type A, $u_{B}\left(q_{m}\right)$ uncertainty Type B.

To determine Type A uncertainty, the probability distribution of values of the observations was examined. Most frequently normal distribution is assumed 'a priori' (especially when the number of measurements is greater than 30).

The estimate of the mass flow was determined as [1]:

$$
\overline{q_{m}}=\frac{1}{n} \sum_{i=1}^{n} q_{m i}
$$

The standard deviation $s$ of an observation was determined from the dependency:

$$
s=\sqrt{\frac{1}{(n-1)} \sum_{i=1}^{n}\left(q_{m i}-\overline{q_{m}}\right)^{2}}
$$


The standard uncertainty $u_{A}\left(q_{m}\right)$ of the measurement is evaluated as [1]:

$$
u_{A}\left(q_{m}\right)=\frac{s}{\sqrt{n}}
$$

Assuming no correlation between the uncertainties of measured quantities, according to the law of the uncertainty propagation [1], the one of Type B determining the mass flow $q_{\mathrm{m}}$ is defined as follows:

$$
\begin{aligned}
& u_{B}\left(q_{m}\right)=\sqrt{\begin{array}{l}
c_{1}{ }^{2} u^{2}(C)+c_{2}{ }^{2} u^{2}(d)+ \\
c_{3}{ }^{2} u^{2}(D)+c_{4}{ }^{2} u^{2}(\Delta p)+ \\
c_{5}{ }^{2} u^{2}\left(\rho_{1}\right)
\end{array}} \\
& =\sqrt{\left(\frac{\partial q_{m}}{\partial C}\right)^{2} u^{2}(C)+\left(\frac{\partial q_{m}}{\partial d}\right)^{2} u^{2}(d)+} \\
& \left(\frac{\partial q_{m}}{\partial D}\right)^{2} u^{2}(D)+\left(\frac{\partial q_{m}}{\partial \Delta p}\right)^{2} u^{2}(\Delta p)+ \\
& \left(\frac{\partial q_{m}}{\partial \rho_{1}}\right)^{2} u^{2}\left(\rho_{1}\right)
\end{aligned}
$$

where:

$c_{1}$ to $c_{5}-$ sensitivity coefficients,

$u_{\mathrm{B}}\left(q_{\mathrm{m}}\right)$ - the estimated uncertainty of measurement of the mass flow $q_{\mathrm{m}}$ with the Type B method,

$u(C)$ - the uncertainty in the discharge coefficient $C$, $u(d)$ - the uncertainty of orifice diameter measurement, $u(D)$ - the uncertainty of pipe diameter measurement, $u(\Delta p)$ - the uncertainty of the differential pressure measurement,

$u\left(\rho_{1}\right)$ - the uncertainty of the density of medium measurement.

In order to estimate the uncertainty of Type B, the equations describing the sensitivity coefficients appearing in equation (8) were established first. Following that the values of sensitivity coefficients are summarized in Table 1 along with their units.

The weight coefficients in equation (8) (partial derivatives) were determined and are also included in Table 1.

The next step of the uncertainty analysis was to estimate the following variances:

a) discharge coefficient $C-u^{2}(C)$,

b) orifice diameter $u^{2}(d)$,

c) pipe diameter $u^{2}(D)$,

d) differential pressure $u^{2}(\Delta p)$,

e) density of a medium $u^{2}\left(\rho_{1}\right)$.

The variance of the discharge coefficient $u^{2}(C)$ was estimated on the assumption of the normal probability distribution for estimation of the discharge coefficient $C$, as:

$$
u^{2}(C)=\left(\frac{\Delta C}{2}\right)^{2}
$$

where $\Delta C$ is the estimation error of the coefficient $C$.
Table 1. The weight coefficients arranged to equation (8) (partial derivatives).

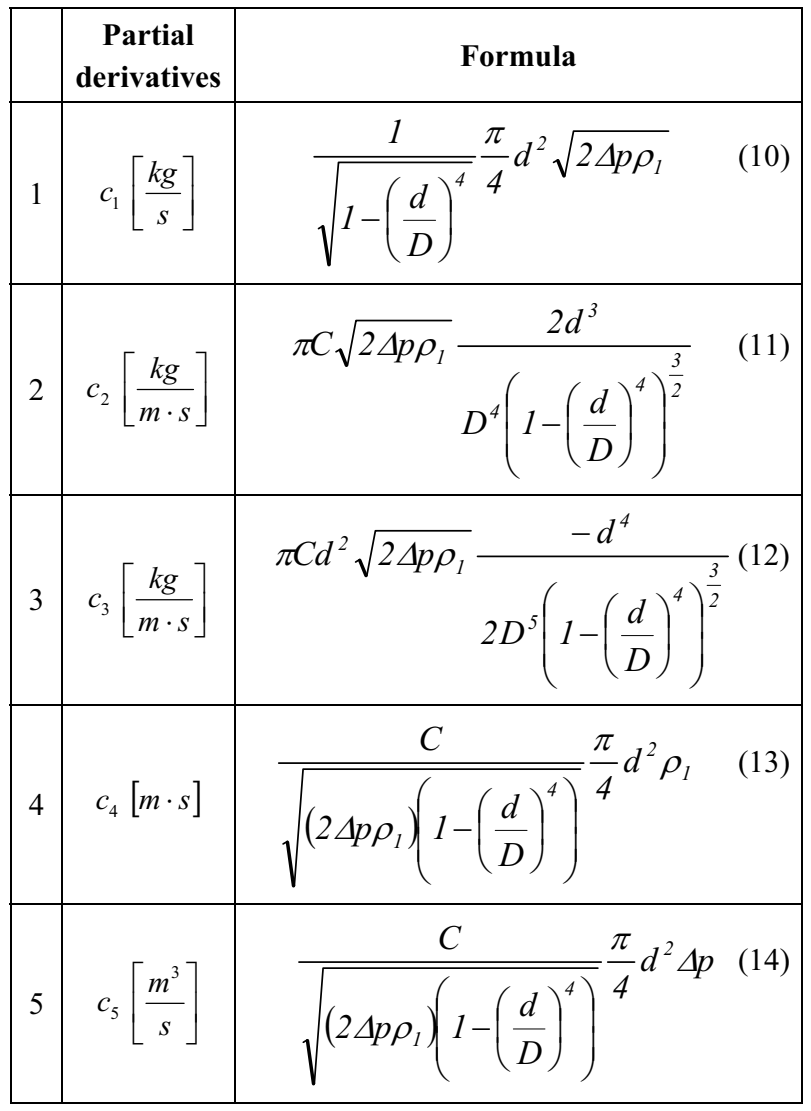

Consequently the variances of the orifice diameter $u^{2}(d)$ and pipe diameter $u^{2}(D)$ were estimated on the assumption of the rectangular probability distribution of the orifice $d$ and pipe $D$ diameters tolerances, as:

$$
\begin{aligned}
& u^{2}(d)=\left(\frac{\Delta d}{\sqrt{3}}\right)^{2} \\
& u^{2}(D)=\left(\frac{\Delta D}{\sqrt{3}}\right)^{2}
\end{aligned}
$$

where $\Delta d$ and $\Delta D$ are tolerances of the apropriate diameters.

The differential pressure $\Delta p$ is usually measured by differential pressure transducer, which is made with the accuracy $\Delta(\Delta p)$. The differential pressure variance $u^{2}(\Delta p)$, resulting from the processing error at the pressure transducer was determined on the assumption of the rectangular probability distribution, as:

$$
u^{2}(\Delta p)=\left(\frac{\Delta(\Delta p)}{\sqrt{3}}\right)^{2}
$$

The following discussion assumed that density of the medium $\rho_{1}$ is constant. The variance of this density $u^{2}\left(\rho_{1}\right)$ was determined also on the assumption of the rectangular probability distribution, as: 


$$
u^{2}\left(\rho_{1}\right)=\left(\frac{\Delta \rho_{1}}{\sqrt{3}}\right)^{2}
$$

After determining the combined uncertainty $u_{\mathrm{c}}\left(q_{\mathrm{m}}\right)$ according to formula (4), one should calculate the expanded uncertainty $U_{\mathrm{p}}$, which is a measure of the required quality of mass flow $q_{\mathrm{m}}$. The expanded uncertainty $U_{\mathrm{p}}$ is determined following the formula:

$$
U_{p}\left(q_{m}\right)=k_{p} \cdot u_{c}\left(q_{m}\right)
$$

The most commonly accepted value of the coverage factor $k_{\mathrm{p}}$ is equal to 2 for confidence level $p=95 \%$.

However, it is more accurate to determine the coverage factor $k_{\mathrm{p}}=t_{\mathrm{p}}\left(v_{\mathrm{eff}}\right)$ on the adopted probability distribution and the basis of the combined uncertainty values $u_{\mathrm{c}}\left(q_{\mathrm{m}}\right)$, as well as the knowledge concerning the number of degrees of freedom for the uncertainty Type $\mathrm{A}-v_{\mathrm{A}}$ and Type B $-v_{\mathrm{B}}$.

The $v_{\text {eff }}$ signifies effective degrees of freedom and can be obtained from the expanded Welch-Satterthwaite formula [1]:

$$
v_{e f f}=\frac{u_{c}^{4}\left(q_{m}\right)}{\frac{u_{A}^{4}\left(q_{m}\right)}{v_{A}}+\frac{u_{B}^{4}\left(q_{m}\right)}{v_{B}}}
$$
as [1]:

The number of degrees of freedom $v_{\mathrm{A}}$ was designated

$$
v_{A}=n-1
$$

Consequently the number of degrees of freedom $v_{\mathrm{B}}$ can be estimated from the squared relative uncertainty $\delta_{\text {rel,B }}^{2}$ according to the following formula [1]:

$$
v_{B}=\frac{1}{2 \cdot \delta_{r e l, B}^{2}}
$$

The values of $v_{\text {eff }}$ were read out from t-Student's table as corresponding to coverage factors $k_{\mathrm{p}}$.

Those results should be presented as the mass flow estimate $q_{\mathrm{m}}$ with the expanded uncertainty $U_{\mathrm{p}}\left(q_{\mathrm{m}}\right)$ :

$$
\left(q_{m} \pm U_{p}\left(q_{m}\right)\right) \frac{k g}{s}
$$

and with information about the confidence level $p$, the effective degrees of freedom $v_{\text {eff }}$, coverage factor $k_{\mathrm{p}}$ and probability distribution.

\subsection{Analysis of the measurement uncertainty according to Monte Carlo method}

The Monte Carlo numerical method [15] is used for simulations of different kinds of phenomena, it is especially effective in case where the analytical description of the phenomenon is complicated.

This method is a numerical tool which simulates generally an unlimited number of unique measurements by random sampling from the known probability density function of all input quantities and propagates their distributions for the measurement model as the output.

The Monte Carlo procedure is conducted as follows:

1) the number $M$ of the trials should be selected,

2) generate $M$ vectors by random sampling from the probability density function for the (set of $N$ ) input quantities,

3) for each vector, evaluate the model to give the corresponding output quantity,

4) estimate of the output of the model,

5) sort the model values into non-decreasing order,

6) use the sorted values to estimate the uncertainty for the output.

In metrology it is used to verify the estimates of analytical uncertainty, especially in cases when dealing with an indirect measurement of the measured value [1617] and when the measuring function is non-linear.

\section{The example}

In order to better illustrate the methodology presented above for estimating the uncertainty of measurement, it was decided to attach an example of the mass flow $q_{\mathrm{m}}$ measurement. This measurement is carried out as shown in Fig. 1. The following data was adopted Table 2 (measurement conditions are compliant with the ISO Standards [18-19]).

Table 2. Data.

\begin{tabular}{|c|c|c|c|}
\hline & Parameter & Unit & Value \\
\hline 1 & $C$ & {$[-]$} & 0.605070 \\
\hline 2 & $d$ & {$[\mathrm{~m}]$} & 0.073648 \\
\hline 3 & $D$ & {$[\mathrm{~m}]$} & 0.100051 \\
\hline 4 & $\varepsilon$ & {$[-]$} & 1.000000 \\
\hline 5 & $\Delta p$ & {$[\mathrm{~Pa}]$} & 2753.400 \\
\hline 6 & $\rho_{1}$ & {$\left[\mathrm{~kg} / \mathrm{m}^{3}\right]$} & 1.109800 \\
\hline 7 & $\beta$ & {$[-]$} & 0.736100 \\
\hline
\end{tabular}

Assumptions raised in paragraph 2 are still applied. A series of 40 mass flow rate $q_{\mathrm{m}}$ results were obtained and are summarized in Table 3 . An estimated value of the mean flow rate $q_{\mathrm{m}}$ was calculated according to the relation (5), on the basis of which the value of $239.5710^{-}$ ${ }^{3} \mathrm{~kg} / \mathrm{s}$ was reached. 
Table 3. Data of the mass flow $q_{\mathrm{m}}$.

\begin{tabular}{|c|c|c|c|c|c|}
\hline$i$ & $q_{\mathrm{mi}}[\mathrm{kg} / \mathrm{s}]$ & $i$ & $q_{\mathrm{mi}}[\mathrm{kg} / \mathrm{s}]$ & $i$ & $q_{\mathrm{mi}}[\mathrm{kg} / \mathrm{s}]$ \\
\hline 1 & 0.237787 & 14 & 0.238402 & 27 & 0.241761 \\
\hline 2 & 0.238122 & 15 & 0.240914 & 28 & 0.238990 \\
\hline 3 & 0.240419 & 16 & 0.238900 & 29 & 0.239754 \\
\hline 4 & 0.240966 & 17 & 0.240062 & 30 & 0.238643 \\
\hline 5 & 0.241060 & 18 & 0.243598 & 31 & 0.239063 \\
\hline 6 & 0.237631 & 19 & 0.240143 & 32 & 0.239783 \\
\hline 7 & 0.239521 & 20 & 0.238266 & 33 & 0.237751 \\
\hline 8 & 0.239253 & 21 & 0.237290 & 34 & 0.242054 \\
\hline 9 & 0.239305 & 22 & 0.237893 & 35 & 0.238818 \\
\hline 10 & 0.236826 & 23 & 0.241714 & 36 & 0.241229 \\
\hline 11 & 0.238927 & 24 & 0.236430 & 37 & 0.239436 \\
\hline 12 & $0, .238068$ & 25 & 0.240820 & 38 & 0.238690 \\
\hline 13 & $0, .240917$ & 26 & 0.241837 & 39 & 0.241107 \\
\hline & & & & 40 & 0.240567 \\
\hline
\end{tabular}

\subsection{Results of the analysis of the measurement uncertainty according to GUM}

The first step in order to determine the mass flow $q_{\mathrm{m}}$ measurement uncertainty was the choosing of uncertainty Type A. According to the model equation (7) the value $u_{\mathrm{A}}\left(q_{\mathrm{m}}\right)$ equals $0.25 \cdot 10^{-3} \mathrm{~kg} / \mathrm{s}$.

The next step was to estimate the uncertainty of Type $\mathrm{B}$, basing on the sensitivity coefficients appearing in equation (8). Their values are summarized in Table 4.

Table 4. The values of the partial derivatives.

\begin{tabular}{|c|c|c|}
\hline & $\begin{array}{c}\text { Partial } \\
\text { derivatives }\end{array}$ & Value \\
\hline 1 & $\left(\frac{\partial q_{m}}{\partial C}\right)\left[\frac{\mathrm{kg}}{\mathrm{s}}\right]$ & $3.96 \mathrm{E}-01$ \\
\hline 2 & $\left(\frac{\partial q_{m}}{\partial d}\right)\left[\frac{\mathrm{kg}}{\mathrm{m} \cdot \mathrm{s}}\right]$ & $9.22 \mathrm{E}+00$ \\
\hline 3 & $\left(\frac{\partial q_{m}}{\partial D}\right)\left[\frac{\mathrm{kg}}{\mathrm{m} \cdot \mathrm{s}}\right]$ & $-1.99 \mathrm{E}+00$ \\
\hline 4 & $\left(\frac{\partial f}{\partial \Delta p}\right)[\mathrm{m} \cdot \mathrm{s}]$ & $4.35 \mathrm{E}-05$ \\
\hline 5 & $\left(\frac{\partial f}{\partial \rho_{1}}\right)\left[\frac{\mathrm{m}^{3}}{\mathrm{~s}}\right]$ & $1.08 \mathrm{E}-01$ \\
\hline
\end{tabular}

The next step in the estimation of measurement uncertainty was to estimate the following variances; discharge coefficient $C-u^{2}(C)$, orifice diameter $u^{2}(d)$, pipe diameter $u^{2}(D)$, differential pressure $u^{2}(\Delta p)$, and density of the medium $u^{2}\left(\rho_{1}\right)$.
Table 5 shows the values of the variance for the assumed probability distributions and the relative errors $\delta$ of the various parameters measurements.

Table 5. The values of the partial variances.

\begin{tabular}{|c|c|c|c|c|}
\hline & Variance & Error $\delta$ [\%] & Distribution & Value \\
\hline 1 & $u^{2}(C)[-]$ & 0.73 & normal & $4.88 \mathrm{E}-06$ \\
\hline 2 & $u^{2}(d)\left[\mathrm{m}^{2}\right]$ & 1.36 & & $4.11 \mathrm{E}-05$ \\
\cline { 1 - 2 } 3 & $u^{2}(D)\left[\mathrm{m}^{2}\right]$ & 0.50 & & $8.34 \mathrm{E}-08$ \\
\hline 4 & $u^{2}(\Delta p)\left[\mathrm{Pa}^{2}\right]$ & 0.40 & rectangular & $3.30 \mathrm{E}-09$ \\
& & & & $4.04 \mathrm{E}+01$ \\
\hline 5 & $u^{2}\left(\rho_{1}\right)\left[\frac{\mathrm{kg}^{2}}{\mathrm{~m}^{6}}\right]$ & 1.00 & & \\
\hline
\end{tabular}

Finally, Type B uncertainty $u_{\mathrm{B}}\left(q_{\mathrm{m}}\right)$ was $1.39 \cdot 10^{-3} \mathrm{~kg} / \mathrm{s}$, which is more than 5 times greater than the Type A uncertainty $u_{\mathrm{A}}\left(q_{\mathrm{m}}\right)$.

Figure 2 shows the share of particular elements of Type B mass flow uncertainty $u_{\mathrm{B}}\left(q_{\mathrm{m}}\right)$, according to formula (8).

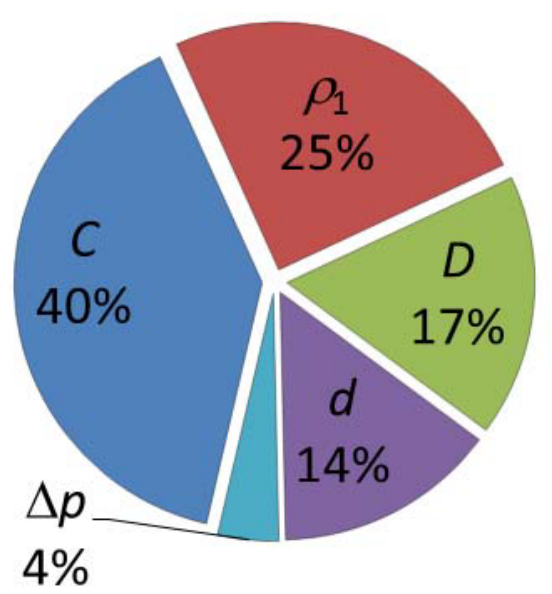

Fig. 2. Share of particular components of the mass flow uncertainty $u_{\mathrm{B}}\left(q_{\mathrm{m}}\right)$.

Summarizing above the most essential uncertainty factor is the discharge coefficient $C$. It brings the greatest information about the measurement precision. Contribution of the rest parameters in the $q_{\mathrm{m}}$ uncertainty in descending order is as follows: density of the medium $\rho_{1}$, pipe diameter $D$ and orifice diameter $d$.

Using the formula (4), the complex uncertainty $u_{\mathrm{c}}\left(q_{\mathrm{m}}\right)$ was $1.41 \cdot 10^{-3} \mathrm{~kg} / \mathrm{s}$.

The last step in estimating the uncertainty of measurement of the mass flow $q_{\mathrm{m}}$ is the calculation of the expanded uncertainty.

The expanded uncertainty of the mass flow $U_{\mathrm{p}}\left(q_{\mathrm{m}}\right)$ measurement for the coverage factor $k_{\mathrm{p}}=2$ (which corresponds to approximately $95 \%$ probability of expansion) is: 


$$
U_{p}\left(q_{m}\right)=2 \cdot u_{c}\left(q_{m}\right)=2.83 \cdot 10^{-3} \frac{\mathrm{kg}}{\mathrm{s}}
$$

On the other hand, assuming coverage factor $k_{\mathrm{p}}=t_{\mathrm{p}}\left(v_{\mathrm{eff}}\right)=2.01$, and that the relative uncertainty $\delta_{\text {rel, } \mathrm{B}}$ of estimated uncertainty Type B is $10 \%$, the estimated at the same probability the expanded uncertainty of the mass flow $U_{\mathrm{p}}\left(q_{\mathrm{m}}\right)$ measurement is $2.88 \cdot 10^{-3} \mathrm{~kg} / \mathrm{s}$.

Table 6 shows the uncertainty values collected during the mass flow estimation on base of the attached data.

\subsection{Results of the analysis of the measurement uncertainty according to Monte Carlo method}

In order to verify results of the estimated uncertainty obtained in the previous section, the Monte Carlo simulaton was performed [9]. This approach is based on the definition of the range expansion, and limits of them were defined by quantile of the probability distribution associated with the measured values.

For this purpose, the random number generator from Microsoft Excel was used. It is assumend that the function of measuring mass flow, according to equation (3) is:

Table 6. Uncertainty budget of mass flow $q_{\mathrm{m}}$ estimate.

\begin{tabular}{|c|c|c|c|c|c|}
\hline $\begin{array}{c}\text { Source of } \\
\text { uncertainty }\end{array}$ & Value & Standard uncertainty & $\begin{array}{l}\text { Probability } \\
\text { distribution }\end{array}$ & \begin{tabular}{|c|} 
Sensitivity coefficient \\
$c_{n}$ \\
\end{tabular} & Variance \\
\hline$q_{m}$ & 0.2396 & $2.54 \mathrm{E}-04$ & t-Student & 1 & $6.45 \mathrm{E}-08$ \\
\hline$\Delta C$ & 0 & $2.21 \mathrm{E}-03$ & normal & $3.96 \mathrm{E}-01$ & $7.66 \mathrm{E}-07$ \\
\hline$\Delta d$ & 0 & $5.74 \mathrm{E}-05$ & \multirow{4}{*}{ rectangular } & $9.22 \mathrm{E}+00$ & $2.80 \mathrm{E}-07$ \\
\hline$\Delta D$ & 0 & $2.89 \mathrm{E}-04$ & & $-1.99 \mathrm{E}+00$ & $3.31 \mathrm{E}-07$ \\
\hline$\Delta(\Delta p)$ & 0 & $6.36 \mathrm{E}+00$ & & $4.35 \mathrm{E}-05$ & $7.67 \mathrm{E}-08$ \\
\hline$\Delta \rho_{l}$ & 0 & $6.41 \mathrm{E}-03$ & & $1.08 \mathrm{E}-01$ & $4.79 \mathrm{E}-07$ \\
\hline & & & \multicolumn{2}{|c|}{ Standard uncertainty $u_{c}\left(q_{\mathrm{m}}\right)$} & $1.41 \mathrm{E}-03$ \\
\hline & & & \multicolumn{2}{|c|}{ Expanded uncertainty $U_{\mathrm{p}}\left(q_{\mathrm{m}}\right)$} & $2.83 \mathrm{E}-03$ \\
\hline
\end{tabular}

In summary, by using the analytical method in accordance with the guidelines contained in [1] for the case under consideration, the following results were obtained:

$$
(239.57 \pm 2.83) \cdot 10^{-3} \frac{\mathrm{kg}}{\mathrm{s}},
$$

for $p=95 \%$, expansion coefficient, respectively $k_{\mathrm{p}}=2$ and

$$
(239.57 \pm 2.88) \cdot 10^{-3} \frac{\mathrm{kg}}{\mathrm{s}},
$$

for $k_{\mathrm{p}}=t_{\mathrm{p}}\left(v_{\mathrm{eff}}\right)$ and $\mathrm{t}-$ Student distribution.

A greater value of the uncertainty of measurement of the mass flow $q_{\mathrm{m}}$ was achieved when the coverage factor $k_{\mathrm{p}}$ was calculated according to the formula (20) - that is, the range in which $95 \%$ of the measurement results are wider.

The relative uncertainty of the mass flow $q_{\mathrm{m}}$ measurement in the analyzed example, did not exceed $0.5 \%$, however this value is highly dependent on the relative uncertainty $\delta_{\text {rel,B }}$.

$$
\begin{aligned}
& q_{m}=\overline{q_{m}}+c_{0} u_{A}\left(q_{m}\right)+c_{1} u(C)+c_{2} u(d)+ \\
& +c_{3} u(D)+c_{4} u(\Delta p)+c_{5} u\left(\rho_{1}\right)
\end{aligned}
$$

where $c_{1}$ to $c_{5}$ marks sensitivity coefficients, and the coefficient $c_{0}$ is equal to 1 . The rest of the coefficients are defined in Table 1, so their values for the considered example are reported in Table 4.

Following obove the probability distributions for the input quantities were assumed : for discharge coefficient $C$ the normal distribution, while for other parameters: $d$, $D, \Delta p, \rho_{1}$ the rectangular distributions.

Estimation of uncertainty using Monte Carlo was performed in Microsoft Excel for the number of samples $M$ equal to $10^{4}$. The value of the expected mass flow $q_{\mathrm{m}}$ and its density function for a confidence level $p=95 \%$ and $k_{\mathrm{p}}=t_{\mathrm{p}}\left(v_{\mathrm{eff}}\right)=2.01$ was determined and applied to the following presentation.

Figure 3 shows the probability density function of the simulated numerical values of the mass flow $q_{\mathrm{m}}$. Based on these results a histogram established with channel $0.4 \cdot 10^{-3} \mathrm{~kg} / \mathrm{s}$, was plotted in Fig. 4. 


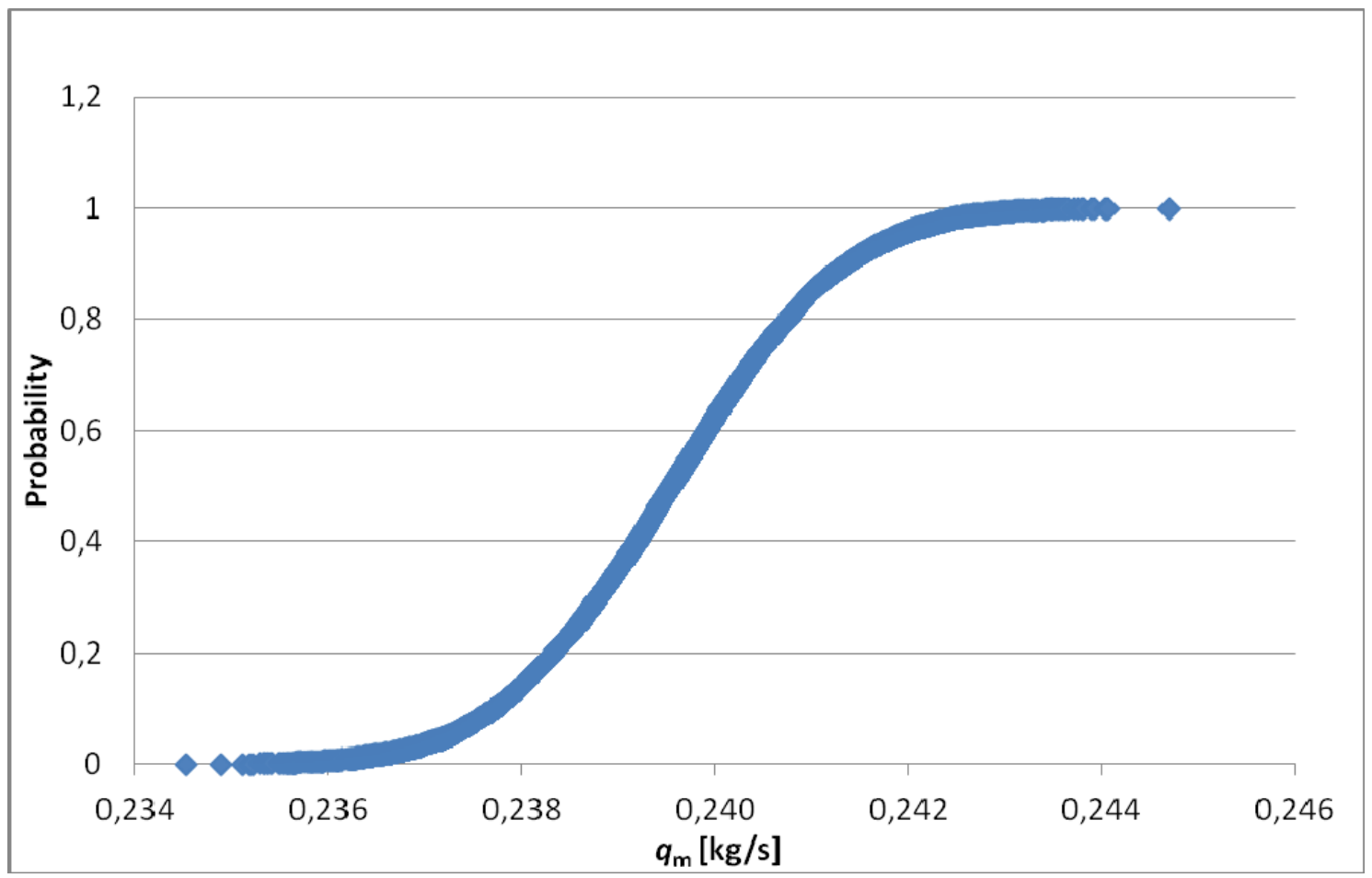

Fig. 3. Simulated the outflow distribution of the $q_{\mathrm{m}}$.

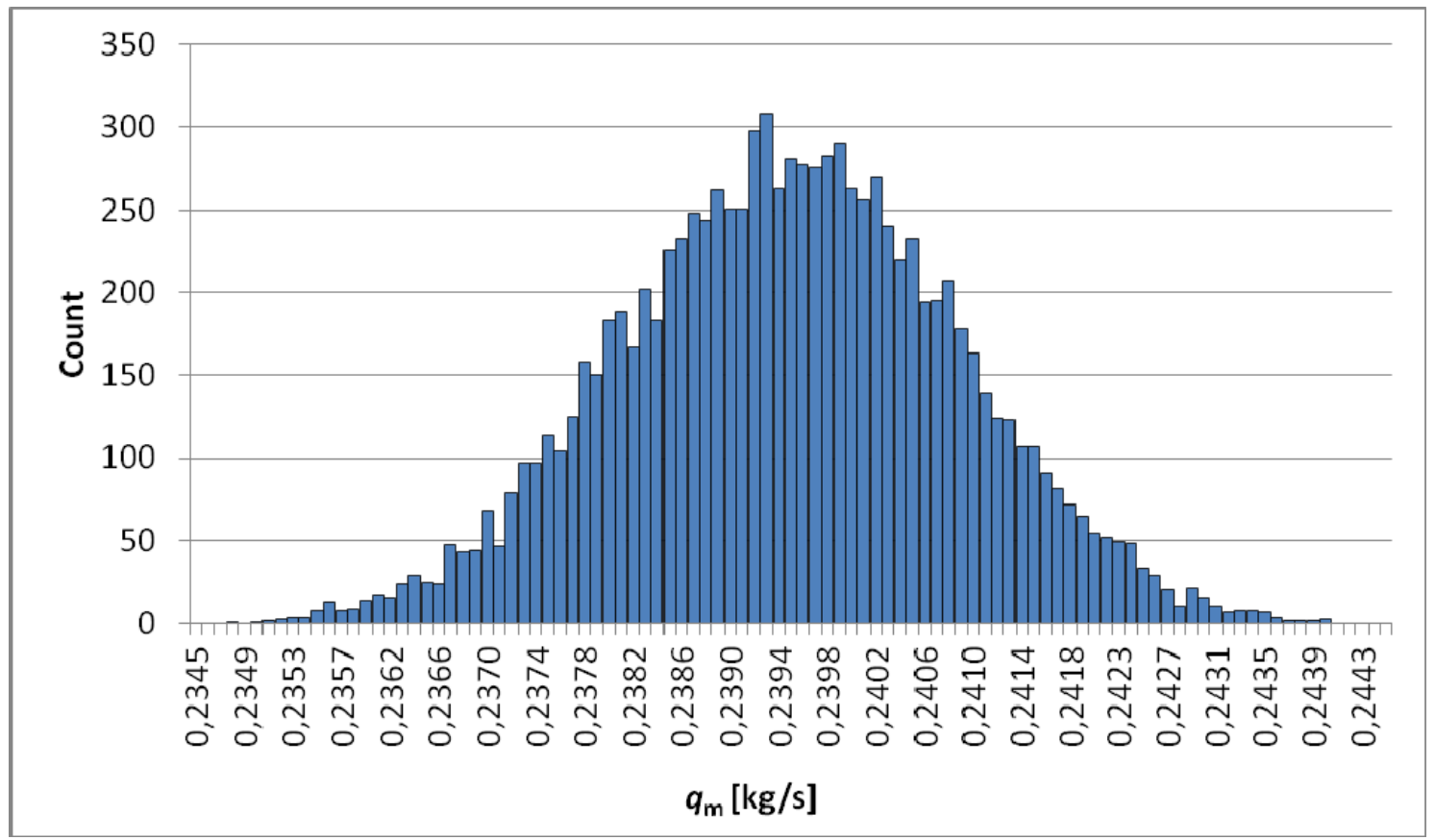

Fig. 4. Histogram for $q_{\mathrm{m}}$.

Table 7 summarizes the results obtained for estimation of value of mass flow $q_{m}$ and the expanded uncertainty $U_{\mathrm{p}}\left(q_{\mathrm{m}}\right)$ estimated with both; the method of propagation of uncertainties (traditional one), and the Monte Carlo one.

It can be observed that the results of measurement uncertainty of the mass flow by both methods are similar. The relative uncertainty is equal to, respectively: $1.20 \%$ - by the traditional method, and $1.15 \%$ by the Monte Carlo one.
Table 7. The expanded standard uncertainty of the mass flow.

\begin{tabular}{|c|c|c|c|}
\hline \multicolumn{4}{|c|}{ Mass flow estimation $\boldsymbol{q}_{\mathbf{m}}$} \\
\hline$p$ & $k_{p}$ & Traditional method & Monte Carlo \\
$10^{-3}[\mathrm{~kg} / \mathrm{s}]$ & $10^{-3}[\mathrm{~kg} / \mathrm{s}]$ \\
\hline 95 & 2.01 & $(239.57 \pm 2.88)$ & $(239.54 \pm 2.77)$ \\
\hline
\end{tabular}




\section{Summary}

The article presents the problem of the mass flow uncertainty estimation using two methods: the method based on the GUM Guide using the law of propagation of uncertainty and the Monte Carlo numerical method.

The authors presented a comprehensive methodology for estimating uncertainty of measurement of the fluid flow through the orifice measurement and calculations carried out for the sample data. The results obtained using the traditional method have been verified by numerical Monte Carlo, and obtained by the one convergence of the estimated measurement uncertainty; those confirmed the correct determination of the analytical method.

The uncertainty of estimating methodology described in the paper can be adapted for use in the metering stations with free selected parameters not only those presented in the article.

The article shows a procedure for estimating uncertainty of the liquid mass flow measurement using the orifice plate. All results refer to a specific case, but the used methodology can be easy extended to other methods.

Standard estimation of the measurements uncertainty is essential in scientific research. Fulfill these conditions allow the assessment of measurements made in different institutions and allow the experimental verification of announced hypotheses in any place in the world.

\section{References}

1. Guide to the Expression of Uncertainty in Measurement (JCGM 100 2008).

2. Handbook of uncertainty calculations fiscal orifice gas and turbine oil metering stations http://nfogm.no/wp-content/uploads/2014/04/ Handbook-USM-fiscal-gas-metering-stations.pdf

3. A. Golijanek-Jędrzejczyk, Przegl. Elektrotech. 85 (2), 25-28 (2009) (in Polish)

4. A. Golijanek-Jędrzejczyk, MAM 58 (11), 987-990 (2012)

5. A. Dzwonkowski, A. Golijanek-Jędrzejczyk, L. Rafiński, Zeszyty Naukowe Wydziału
Elektrotechniki i Automatyki Politechniki Gdańskiej 34, 21-24 (2013) (in Polish)

6. A. Dzwonkowski, A. Golijanek-Jędrzejczyk, Przegl. Elektrotech. 91 (10), 166-169 (2015)

7. A. Dzwonkowski, MAM 59 (5), 394-397 (2013)

8. R.C. Baker, An introductory guide to flow measurement (Alden Press, Oxford, 1989)

9. JP. De Carlo, Fundamentals of flow measurement Instrument Society of America (Research Triangle Park, NC, 1984)

10. E.L. Upp, P.J. LaNasa, Fluid Flow Measurement; a Practical Guide to Accurate Flow Measurement, (Gulf Professional Publishing, MA, 2002)

11. M. Reader-Harris, Orifice Plates and Venturi Tubes, (Springer International Publishing, Switzerland, 2015), DOI 10.1007/978-3-31916880-7_2

12. A. Harrouz, O. Harrouz, A. Benatiallah, IJPEDS 3(4), 450-468 (2013)

13. L. Petryka, M. Zych, R. Hanus, J. Sobota, P.Vlasak, M. Sleziak, D. Świsulski, EPJ WoC 114, 02093 (2016)

14. http://www.ematem.org/Dokumente/2008_lau_calc ulat.pdf

15. Supplement 1 to the Guide to the expression of uncertainty in measurement - Propagation of distributions using a Monte Carlo method (JCGM $101: 2008)$

16. P. Fotowicz, Pomiary Automatyka Robotyka 1/2005, 5-9 (2005)

17. A. Dzwonkowski, A. Golijanek-Jędrzejczyk, L. Rafiński, Przegl. Elektrotech. 91 (8), 11-14 (2015)

18. ISO Measurement of fluid flow by means of pressure differential devices inserted in circular cross-section conduits running full-Part 1: general principles and requirements. (International Organization for Standardization, Geneva, ISO 5167-1:2003)

19. ISO Measurement of fluid flow by means of pressure differential devices inserted in circular cross-section conduits running full-Part 2: Orifice plates. (International Organization for Standardization, Geneva, ISO 5167-2:2003) 